\title{
Asian flushing presents opportunities for disease prevention
}

\author{
Friedrich C. Luft $^{1}$
}

Published online: 6 September 2016

(C) Springer-Verlag Berlin Heidelberg 2016

The aldehyde dehydrogenases are a family of detoxifying enzymes that oxidize aldehydes to carboxylic acids. ALDH1 and ALDH2 are the most important enzymes for aldehyde oxidation, and both are tetrameric enzymes composed of $54 \mathrm{kD}$ subunits. These enzymes are found in many tissues of the body but are at the highest concentration in the liver. The overall reaction catalyzed by the aldehyde dehydrogenases is the following: $\mathrm{R}-\mathrm{CHO}+\mathrm{NAD}^{+}+\mathrm{H}_{2} \mathrm{O} \rightarrow \mathrm{R}-\mathrm{COOH}+\mathrm{NADH}$ $+\mathrm{H}^{+}$. The reaction is NAD $(\mathrm{P})^{+}$-dependent. The aldehyde enters the enzyme's active site through a channel located outside of the enzyme. A sulfur atom from a cysteine in the active site attacks the carbonyl carbon of the aldehyde. The hydrogen is kicked off as a hydride and in turn attacks $\mathrm{NAD}(\mathrm{P})^{+}$to make $\mathrm{NAD}(\mathrm{P}) \mathrm{H}$. The enzyme's active site then undergoes an isomorphic change whereby the $\mathrm{NAD}(\mathrm{P}) \mathrm{H}$ is moved, allowing a water molecule to access the substrate. A glutamate primes the water in the active site and the water conducts a nucleophilic attack on the carbonyl carbon, kicking off the sulfur.

The human genome has 19 ALDH genes. However, one ALDH emerges as a particularly important enzyme in a variety of human conditions. This ALDH is ALDH2, located in the mitochondrial matrix. Much is known about ALDH2's role in ethanol metabolism [1]. Less appreciated is the fact that ALDH2 dysfunction may contribute to a variety of human diseases including heart disease, neurodegenerative disorders, stroke, cancer, and diabetes mellitus. An ALDH2 inactivating mutation (termed ALDH2*2; Glu504Lys) is the most common single point mutation in humans. Epidemiological

Friedrich C. Luft

friedrich.luft@charite.de

1 Experimental and Clinical Research Center, Max-Delbrück Center for Molecular Medicine and Charité Medical Faculty, Lindenberger Weg 80, 13125 Berlin, Germany studies suggest a correlation between this inactivating mutation and increased propensity for human diseases. The allele is found in as many as 35-45\% of East Asians, namely Chinese, Japanese, Korean, and Taiwanese peoples [2]. The ALDH $2 * 2$ carriers have a lower ALDH2 enzymatic activity. The relative deficiency is responsible for facial flushing, headaches, nausea, dizziness, and cardiac palpitations after alcohol consumption. The ALDH $2 * 2$ polymorphism also contributes to variation in efficacy of sublingual nitroglycerin [3]. For allele carriers, the symptoms are a convenient "stopping signal" after alcohol ingestion. Thus, far, disulfiram is the only United States Food and Drug Administration (FDA)-approved drug that effectively targets ALDH2. Disulfiram and its metabolites irreversibly inactivate catalytic Cys302 in ALDH2 by carbamylation in the enzyme's substrate site [4].

However, the relevance of ALDH2 extends far beyond alcohol or its abuse. ALDH2 has rapidly emerged as a crucial enzyme involved in protecting the heart from oxidative stress [5]. The cardioprotective effects of ALDH2 have also been highlighted by epidemiological studies, demonstrating that individuals with ALDH2*2 are more susceptible to cardiac diseases [6]. The generalized role of ALDH2 in human chronic disease has also been verified in animal studies and human pathological findings. The conditions include particularly Parkinson's disease and Alzheimer's dementia [1]. However, relevant to this discussion is a connection between ALDH2 and diabetes mellitus. Since Asian countries are predicted to make up $60 \%$ of the world's diabetic population in the near future, the identification of ALDH $2 * 2$ as a risk factor for type 2 diabetes mellitus is a highly significant observation.

How the association between ALDH2 and type 2 diabetes was identified is interesting. Clinicians observed that chlorpropamide, a sulfonyl urea drug used to treat type 2 diabetes, was observed to elicit facial flushing in patients [7]. Chlorpropamide inhibits ALDH, suggesting the possible link 
between ALDH and diabetes. Furthermore, persons enjoying more than 20 drinks per week commonly develop insulin resistance; those harboring ALDH $2 * 2$ require far fewer drinks per week [8]. Earlier studies have shown that transgenic mice overexpressing of ALDH2, are protected from streptozotocininduced diabetic cardiomyopathy. In this setting of diabetic complications, Alda-1, an ALDH2 activator, was able to recapture the protective effect against high glucose-induced mitochondrial damage and contractile function in cardiomyocytes [9].

The fact that aldehydes are toxic has been known for a long time. The molecules diffuse through cell membranes, form adducts with macromolecules including proteins, DNA, and lipids. Such aldehyde adduction disrupts the function of these macromolecules. With diminished ALDH activity, the disruption is enhanced. Furthermore, increased oxidative stress causes a build-up of toxic aldehydes such as 4hydroxynonenal (4-HNE) that promote cell death. 4-HNE is an $\alpha, \beta$-unsaturated hydroxyl alkenal that is produced by lipid peroxidation. 4-HNE has three reactive groups, namely an aldehyde, a double bond at carbon 2 , and a hydroxy group at carbon 4. ALDH2 metabolizes toxic aldehydes such as 4HNE [10].

In this issue of J Mol Med, Wang et al. show that ALDH2 deficiency disturbs energy metabolism and diastolic cardiac function in diabetic mice [11]. The authors relied on ALDH2 gene-deleted mice, compared to normal littermates. The mice were then subjected to streptozotocin or sham injection, forming four groups. At 4 weeks, there were no significant changes observed in myocardial histology or ultrastructure. Nevertheless, functional studies revealed that although systolic function was maintained, diastolic function was impaired. When the authors examined energy metabolism in their mice, they found that energy metabolism was impaired in diabetic ALDH2 - /- mice compared to the other groups. The authors next observed that diabetes-induced oxidative stress and AMP-activated protein kinase (AMPK) phosphorylation were increased. A detailed metabolomics analysis indicated that diabetic ALDH2 - - mice had dysregulated glucose homeostasis, purine nucleotide metabolism, and activation of endogenous protective responses. Various phospholipid metabolites suggested that phospholipid metabolism was perturbed. Interestingly, 4-HNE expression was increased in diabetic ALDH2-/- mice. Finally, riboflavin, carnitine, L-tyrosine, arachidonic acid, and phospholipid level changes suggested that the diabetes risk in these mice was increased.

Next, the authors recruited 118 subjects with type 2 diabetes from a population-based cohort [11]. Within these subjects they were able to compare ALDH2*2, Glu504Lys, (GG), and ALDH2 (GA-AA). Not sufficient (AA) subjects were available to allow a separate analysis for homozygous AA persons. In this small association study, the authors found that the (GAAA) alleles were associated with impaired diastolic but not systolic cardiac function.

Although, perhaps protected from excess alcohol consumption, the findings suggest that ALDH2*2 could be unhealthy, particularly in the type 2 diabetes-prone environment. However, help could be on the way. Chen et al. have developed ALDH activators termed Aldas [5]. Alda-1 [N-(1,3benzodioxol-5-ylmethyl)-2,6-dichlorobenzamide, MW 324] is an agonist that enhances the catalytic activity of ALDH2 in vitro and in vivo. Alda- 1 increases the catalytic activity of ALDH2*2 homozygotic enzyme by 11-fold and brings the activity of the heterozygous enzyme to wild-type levels [5]. Co-crystal structures of Alda- 1 with wild-type ALDH2 and Alda- 1 with the E487K mutant ALDH2 demonstrated that Alda-1 binds at the
Fig. 1 Alda-1, an allosteric agonist of ALDH2, corrects the structural defect in the ALDH2*2 mutant present in $8 \%$ of the human population (from Chen et al. [1] with permission). Upper panel shows a stick presentation with the structure of Alda-1 in the catalytic tunnel. The critical amino acids are highlighted. Lower panel shows crystal structure of wild-type ALDH2 and mutant ALDH2*2. $\mathrm{ALDH} 2 * 2$ is restored when bound with Alda-1
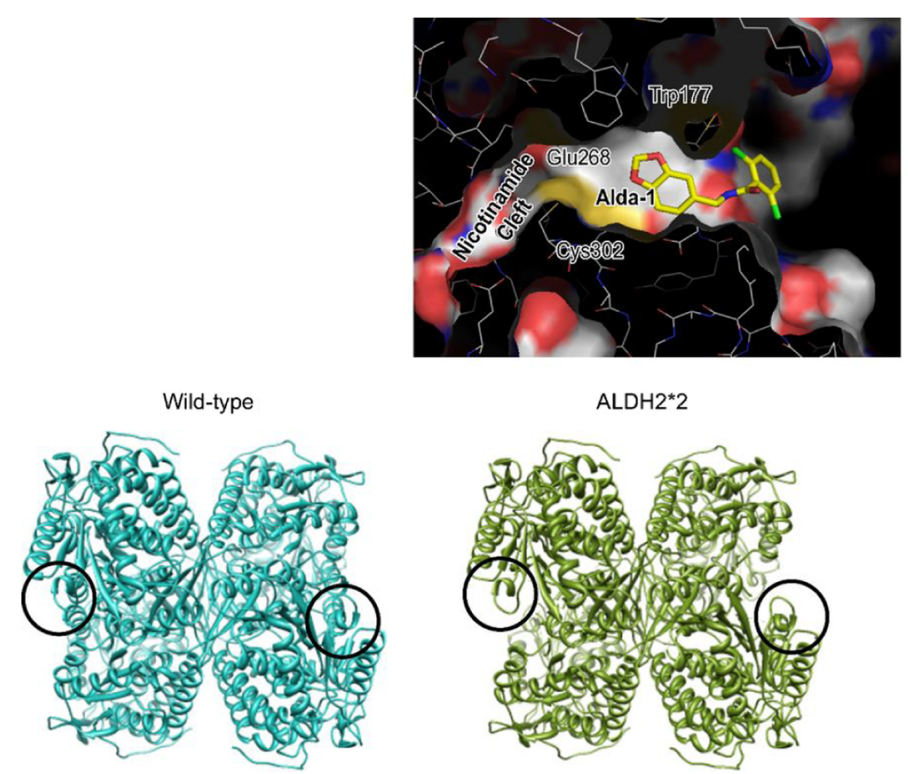

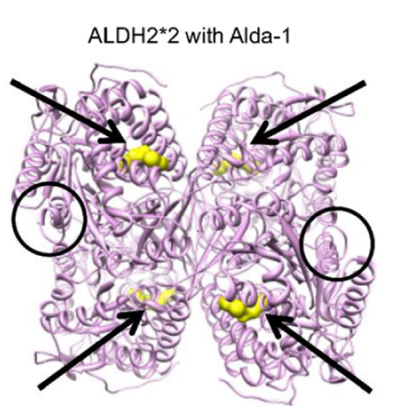


entrance of the catalytic tunnel in close proximity to Cys302 and Glu286, which are critical to its substrate catalysis (Fig. 1) [12].

Alda- 1 and analogs are a remarkable development given the broad scope of diseases, including cardiovascular, metabolic, neurodegenerative, musculoskeletal, and malignant diseases that are influenced by this single example of an ALDH. There are 18 other family members perhaps also of medical importance. These enzymes represent a shield from oxidative stress and aldehydic loads. While the ALDH $2 * 2$ mutation is regarded as being of relatively minor importance, the data presented here suggest that "Asian flushing" could have much greater consequences than believed. Whether or not ALDH2 activators such as Alda-1 will slow down the progression of human diseases or play a preventative role will require much experimental and clinical work. Nevertheless, the topic raises a marked potential for pharmaceutical development. Finally, Alda-1 could enable our Asian friends with $\mathrm{ALDH} 2 * 2$ to raise their glasses; therefore, skål!!!

Respectfully,

Friedrich C. Luft

\section{References}

1. Chen CH, Ferreira JC, Gross ER, Mochly-Rosen D (2014) Targeting aldehyde dehydrogenase 2: new therapeutic opportunities. Phys Rev 94:1-34

2. Dandre F, Cassaigne A, Iron A (1995) The frequency of the mitochondrial aldehyde dehydrogenase i2 (atypical) allele in caucasian, oriental and african black populations determined by the restriction profile of pcr-amplified DNA. Mol Cell Probes 9:189-193
3. Li Y, Zhang D, Jin W, Shao C, Yan P, Xu C, Sheng H, Liu Y, Yu J, Xie Y, et al. (2006) Mitochondrial aldehyde dehydrogenase-2 (aldh2) glu504lys polymorphism contributes to the variation in efficacy of sublingual nitroglycerin. J Clin Invest 116:506-511

4. Lipsky JJ, Shen ML, Naylor S (2001) In vivo inhibition of aldehyde dehydrogenase by disulfiram. Chem Biol Interact:130-132 93-102

5. Chen CH, Budas GR, Churchill EN, Disatnik MH, Hurley TD, Mochly-Rosen D (2008) Activation of aldehyde dehydrogenase-2 reduces ischemic damage to the heart. Science 321:1493-1495

6. Bian Y, Chen YG, Xu F, Xue L, Ji WQ, Zhang Y (2010) The polymorphism in aldehyde dehydrogenase-2 gene is associated with elevated plasma levels of high-sensitivity c-reactive protein in the early phase of myocardial infarction. Tohoku J Exp Med 221:107-112

7. Leslie RD, Pyke DA (1978) Chlorpropamide-alcohol flushing: a dominantly inherited trait associated with diabetes. Br Med J 2: $1519-1521$

8. Jung JG, Kim JS, MK O (2010) The role of the flushing response in the relationship between alcohol consumption and insulin resistance. Alcohol Clin Exp Res 34:1699-1704

9. Zhang Y, Babcock SA, Hu N, Maris JR, Wang H, Ren J (2012) Mitochondrial aldehyde dehydrogenase (aldh2) protects against streptozotocin-induced diabetic cardiomyopathy: role of GSK3beta and mitochondrial function. BMC Med 10:40

10. Guo JM, Liu AJ, Zang P, Dong WZ, Ying L, Wang W, Xu P, Song XR, Cai J, Zhang SQ, et al. (2013) Aldh2 protects against stroke by clearing 4-HNE. Cell Res 23:915-930

11. Wang C, Fan F, Cao Q, Shen C, Zhu H, Wang P, Zhao X, Sun X, Dong Z, Ma X, et al. (2016) Mitochondrial aldehyde dehydrogenase 2 deficiency aggravates energy metabolism disturbance and diastolic dysfunction in diabetic mice. $\mathrm{J}$ Mol Med. doi:10.1007/s00109-016-1449-5

12. Perez-Miller S, Younus H, Vanam R, Chen CH, Mochly-Rosen D, Hurley TD (2010) Alda-1 is an agonist and chemical chaperone for the common human aldehyde dehydrogenase 2 variant. Nat Struct Mol Biol 17:159-164 